\title{
Analysis on Eccentric Bus-bar Testing Method of Heavy Current Transformer
}

\author{
Jin Xing, Wei Zhao, Songling Huang \\ State Key Lab of Power System, Dept. of Electrical \\ Engineering, Tsinghua University \\ Beijing, China \\ xing-j09@mails.tsinghua.edu.cn
}

\author{
Bo Jiang \\ Sichuan Electric Power Test and Research Institute, \\ Chengdu, China
}

\begin{abstract}
For testing the reliability of heavy current transformer (HCTs) with shielding coils affected by the adjacent bus bars, an equivalent testing method called eccentric bus-bar testing method was presented in [1]. The crucial parameters of this method were also determined by analytical formulas and further verified by finite element method (FEM) in [1]. This paper presents several experiments and analytical formulas on this testing method, which reveal a basic flaw of this method. Furthermore, the practical applicability of this equivalent testing method is analyzed.
\end{abstract}

Keywords-heavy current transformer; testing method; eccentric bus-bar; electromagnetic interference

\section{INTRODUCTION}

The heavy current transformers (HCTs) have been widely used in power systems to measure the heavy currents. The HCTs we researched on is mounted on the bus bars at the outset of generators. Usually, there are six bus bars and each at least equipped a HCT for measuring. Therefore, the working state of the HCT will be severely interfered by the adjacent bus bars. Fig. 1 schematically shows the HCT mounted on the bus bar (phase B) and affected by the other five adjacent bus bars.

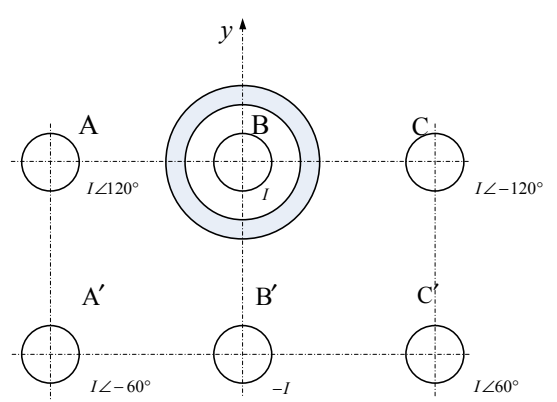

Figure 1. Schematical show of the HCT mounted on the phase B at the outset of a generator

Therefore, testing method is required in order to evaluate the reliability of all this kind of HCTs under such conditions before installing. However, it is true that HCT manufacturers usually cannot supply so heavy and so many bus bar currents even in laboratories, so the equivalent testing methods have been used in practice.

Reference [1] presented a simple equivalent testing method, which only uses one eccentric primary bus bar to simultaneously simulate the effects of the adjacent and primary bus bars. The method is schematically shown in Fig. 2. Reference [1] also determined the crucial parameters of this method by analytical formulas and further verified by finite element method (FEM).

This paper will analyze the eccentric bus-bar testing method by experiment investigations and analytical formulas. The experimental and calculating results are valuable to evaluate this equivalent testing method.

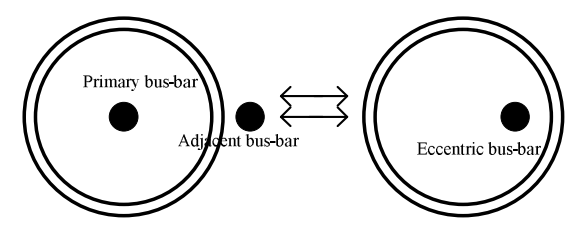

Figure 2. Schematical show of the eccentric bus-bar testing method

\section{EXPERIMENTAL INVESTIGATIONON ON THE TESTING METHOD}

For the purpose of evaluating the eccentric bus-bar testing method and the correctness of the crucial parameters, three experiments (Experiment A, B, C) have been made according to Fig. 3. The experimental HCT $\left(\mathrm{T}_{\mathrm{e}}\right)$ is made up of mumetal with a rated ratio of $5000 / 5 \mathrm{~A}$ and class 0.5 . The inner diameter, outer diameter, and the height of the iron core are $520 \mathrm{~mm}, 560 \mathrm{~mm}$, and $25 \mathrm{~mm}$, respectively. 10-turn search coils are placed each $15^{\circ}$ around the core after winding the evenly distributed secondary winding. For each measure point, 5 times testing were taken to make sure the reliability of the measuring results [2]. The secondary terminals of $T_{e}$ are connected to the electronic load of $30 \mathrm{VA}$ and $\cos \varphi=0.8$. Another HCT $\left(\mathrm{T}_{\mathrm{c}}\right)$ used as a standard current comparator is $5000 / 5$ A with class 0.2 .

Experiment $A$ is $T_{e}$ under normal working conditions without eccentric primary bus bar and the influence of the adjacent bus bar. The primary bus bar flowed through $5000 \mathrm{~A}$ is placed in the center of the core. 


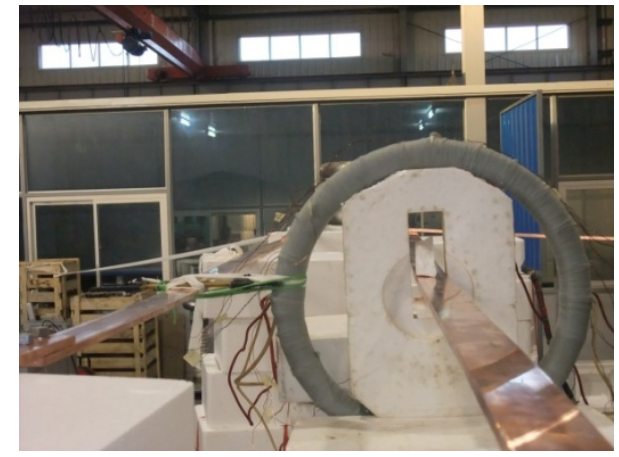

Figure 3. Experimental facility for evaluating the eccentric bus bar testing method and the correctness of the crucial parameters

Experiment B: The normal operating $\mathrm{T}_{\mathrm{e}}$ is affected by the adjacent bus bar which has $5000 \mathrm{~A}$ currents. The distance between the adjacent bus bar and the center of $\mathrm{T}_{\mathrm{e}}$ is $560 \mathrm{~mm}$. From (9) presented in reference [1], the eccentric distance L should be equal to $130 \mathrm{~mm}$ (50\% of the inner radius). The distributions of the magnetic flux density along the circumference of the core under the two conditions are shown in Fig. 4. The current error and the phase displacement are shown in Table I.

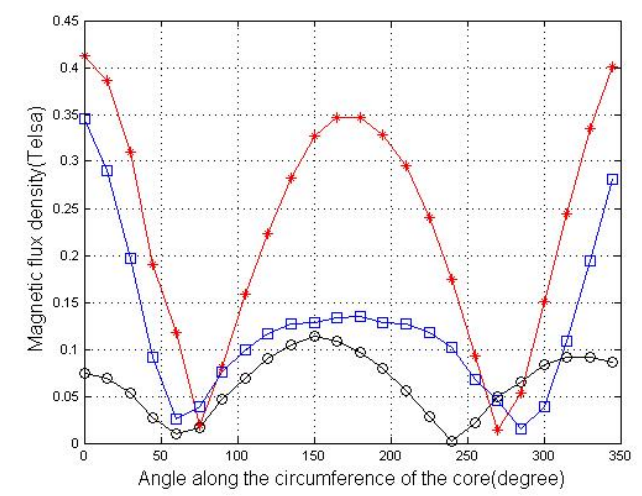

Black curve, magnetic flux density of experiment A

Red curve, magnetic flux density of normal operating Te affected by the adjacent bus bar Blue curve, magnetic flux density of Te with eccentric bus bar

Figure 4. The distributions of the magnetic flux density along the circumference of the core

TABLE I. EXPERIMENTAL ERROR COMPARISON

\begin{tabular}{|l|c|c|c|}
\hline & \multirow{2}{*}{ Experiment A } & \multicolumn{2}{|c|}{ Experiment B } \\
\cline { 3 - 4 } & D=560mm & L=130mm \\
\hline $\begin{array}{l}\text { Current error } \\
\Delta \text { I },(\%)\end{array}$ & -0.217 & -0.351 & -0.309 \\
\hline $\begin{array}{l}\text { Phase } \\
\text { displacement } \\
\delta,(\%)\end{array}$ & +3.7 & +3.5 & +2.9 \\
\hline
\end{tabular}

Experiment $\mathrm{C}$ : The normal operating $\mathrm{T}_{\mathrm{e}}$ is affected by the adjacent bus bar which has $5000 \mathrm{~A}$ currents. The distance between the adjacent bus bar and the center of $\mathrm{T}_{\mathrm{e}}$ is $316.5 \mathrm{~mm}$. From (9) presented in reference [1], the eccentric distance $\mathrm{L}$ is equal to $230 \mathrm{~mm}(88.46 \%$ of the inner radius). The distributions of the magnetic flux density along the circumference of the core under the two conditions are shown in Fig. 5. The current error and the phase displacement are shown in Table II.

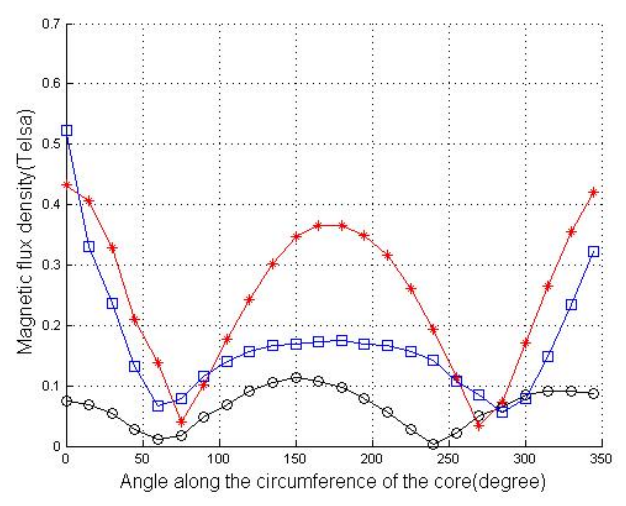

Black curve, magnetic flux density of experiment A

Red curve, magnetic flux density of normal operating Te affected by the adjacent bus bar Blue curve, magnetic flux density of Te with eccentric bus bar

Figure 5. The distributions of the magnetic flux density along the circumference of the core

TABLE II. EXPERIMENTAL ERROR COMPARISON

\begin{tabular}{|l|c|c|c|}
\hline & \multirow{2}{*}{ Experiment A } & \multicolumn{2}{|c|}{ Experiment C } \\
\cline { 3 - 4 } & & $\mathbf{D = 3 1 6 . 5 2 \mathrm { mm }}$ & $\mathbf{L = 2 3 0 \mathrm { mm }}$ \\
\hline $\begin{array}{l}\text { Current error } \\
\Delta \mathrm{I},(\%)\end{array}$ & -0.217 & -0.584 & -0.481 \\
\hline $\begin{array}{l}\text { Phase } \\
\text { displacement } \\
\delta,(\%)\end{array}$ & +3.7 & +3.5 & +4.4 \\
\hline
\end{tabular}

From the Table I and Table II, it can be concluded that the adjacent bus bar and the eccentric primary bus bar would cause nearly the same errors in the measured currents, respectively. And from Fig. 4 and Fig. 5 we can see that the adjacent bus bar and the eccentric primary bus bar would both affect the distributions of the magnetic flux density along the circumference of $T_{e}$ (both making an increase of the flux density near the adjacent bus bar or the eccentric one). However there still have large relative errors between the red curve and the blue one respectively, especially at $0^{\circ}$ and 180 ${ }^{\circ}$. It is required to evaluating the eccentric bus-bar testing method and the correctness of the crucial parameters by analytical formulas to make sure that this equivalent testing method could be used to evaluate the reliability of HCTs.

\section{Evaluating the CRUCial Parameters of THE TESTING METHOD By ANALYTICAL FoRMULAS}

Now we firstly analyze the influence of stray flux on the HCT by analytical formulas. The stray flux produced by the adjacent bus bar enters into the core from point $\mathrm{A}$ and comes out from point B. The flux $\Phi_{B}$ will subtract the stray flux in the left part of the core and add up with the stray flux in the right part, as shown in Fig. 6. The supplementary stray flux will make the flux density unequal in different positions of the core and even cause the saturation phenomena [3]. 


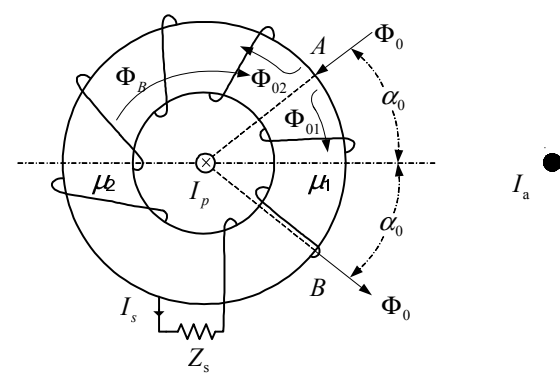

Figure 6. the normal operating HCT affected by the adjacent bus bar

The following assumptions were made: the HCT has not been serious saturated. Then the core of the HCT could be divided into two parts (the left part with the permeability $\mu_{1}$ and the right part with the permeability $\mu_{2}$ ).

According to the assumptions above and Fig. 6, four equations could be got [4]:

The voltage applied on the load $Z_{\mathrm{s}}$ should be equal to the voltage induced by the total flux linking the secondary winding.

$$
I_{\mathrm{s}} Z_{\mathrm{s}}=2 \pi f\left[\frac{2 \alpha_{0}}{2 \pi} N_{\mathrm{s}}\left(\Phi_{\mathrm{B}}+\Phi_{01}\right)+\left(1-\frac{2 \alpha_{0}}{2 \pi}\right) N_{\mathrm{s}}\left(\Phi_{\mathrm{B}}-\Phi_{02}\right)\right]
$$

The total primary ampere turns minus the total secondary ampere turns should be equal to the magnetizing ampere turns of the core.

$$
I_{\mathrm{p}}-I_{\mathrm{s}} N_{\mathrm{s}}=\frac{\frac{2 \alpha_{0}}{2 \pi} l}{A \mu_{1}}\left(\Phi_{\mathrm{B}}+\Phi_{01}\right)+\frac{\left(1-\frac{2 \alpha_{0}}{2 \pi}\right) l}{A \mu_{2}}\left(\Phi_{\mathrm{B}}-\Phi_{02}\right)
$$

The total stray flux in the core should be equal to the flux produced by the adjacent bus bar entering into the core.

$$
\Phi_{01}+\Phi_{02}=\Phi_{0}
$$

Electromagnetic forces (EMF) in the left and right part are equal.

$$
H_{01} l_{1}=H_{02} l_{2}
$$

From (1)-(4), $I_{\mathrm{s}}$ could be solved as,

$$
\begin{gathered}
I_{\mathrm{s}}=\frac{2 \pi f A N_{\mathrm{s}} \mu_{1} \mu_{2}}{Z_{\mathrm{s}} l\left[\mu_{1}-\left(\mu_{1}-\mu_{2}\right) \frac{2 \alpha_{0}}{2 \pi}\right]+N_{\mathrm{s}}^{2} A \mu_{1} \mu_{2}} I_{\mathrm{p}} \\
+\frac{2 \pi f N_{\mathrm{s}}\left(\mu_{1}-\mu_{2}\right) \frac{2 \alpha_{0}}{2 \pi}\left(1-\frac{2 \alpha_{0}}{2 \pi}\right)}{Z_{\mathrm{s}} l\left[\mu_{1}-\left(\mu_{1}-\mu_{2}\right) \frac{2 \alpha_{0}}{2 \pi}\right]+N_{\mathrm{s}}{ }^{2} A \mu_{1} \mu_{2}} \Phi_{0}
\end{gathered}
$$

From (5), we can see that the measurement error of the HCT will be affected by the stray flux mainly in two ways. The first, the stray flux entering into the core directly induces currents in the secondary winding. The second, the stray flux makes the permeability unequal in different positions of the core then changing the secondary currents induced by the primary bus bar [5].

Now we analyze the influence of the eccentric primary bus bar on the HCT. We made the assumption that the primary bus bar had a certain eccentricity which could make the same magnetic flux density in the core with the normal operating HCT influenced by the stray flux, shown in Fig. 7.

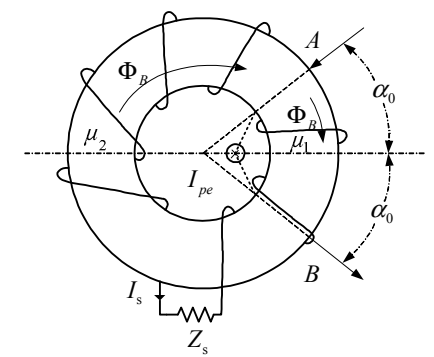

Figure 7. the HCT with the eccentric primary bus bar

According to the assumption and Fig. 7, three equations could be got:

The voltage applied on the load $Z_{\mathrm{s}}$ should be equal to the voltage induced by the total flux linking the secondary winding.

$$
I_{\mathrm{s}} Z_{\mathrm{s}}=2 \pi f N_{\mathrm{s}} \Phi_{\mathrm{B}}
$$

The total primary ampere turns minus the total secondary ampere turns should be equal to the magnetizing ampere turns of the core.

$$
I_{\mathrm{p}}-I_{\mathrm{s}} N_{\mathrm{s}}=\frac{\frac{2 \alpha_{0}}{2 \pi} l}{A \mu_{1}} \Phi_{\mathrm{B}}+\frac{\left(1-\frac{2 \alpha_{0}}{2 \pi}\right) l}{A \mu_{2}} \Phi_{\mathrm{B}}
$$

From (6)-(7), $I_{\mathrm{s}}$ could be solved as

$$
I_{\mathrm{s}}=\frac{2 \pi f A N_{\mathrm{s}} \mu_{1} \mu_{2}}{Z_{\mathrm{s}} l\left[\mu_{1}-\left(\mu_{1}-\mu_{2}\right) \frac{2 \alpha_{0}}{2 \pi}\right]+N_{\mathrm{s}}^{2} A \mu_{1} \mu_{2}} I_{\mathrm{p}}
$$

From (8), it is shown that the eccentric primary bus bar will influence the measurement error of the HCT mainly by changing permeability unequal in different positions of the core.

From (1), this formula could be decomposed to two parts as follows:

$$
\begin{aligned}
I_{\mathrm{s}} Z_{\mathrm{s}}=I_{\mathrm{s}}^{\prime} Z_{\mathrm{s}}^{\prime}+I_{\mathrm{s}}^{\prime \prime} Z_{\mathrm{s}}^{\prime \prime} & \\
=2 \pi f\left[N_{\mathrm{s}} \Phi_{\mathrm{B}}\right]+ & 2 \pi f\left[\frac{2 \alpha_{0}}{2 \pi} N_{\mathrm{s}} \Phi_{01}+\left(1-\frac{2 \alpha_{0}}{2 \pi}\right) N_{\mathrm{s}}\left(-\Phi_{02}\right)\right] \\
I_{\mathrm{p}}-I_{\mathrm{s}} N_{\mathrm{s}} & =\left[\frac{2 \alpha_{0}}{A \pi} l \Phi_{\mathrm{B}}+\frac{\left(1-\frac{2 \alpha_{0}}{2 \pi}\right) l}{A \mu_{2}} \Phi_{\mathrm{B}}\right] \\
& +\left[\frac{\frac{2 \alpha_{0}}{A \pi} l}{A \mu_{1}} \Phi_{01}-\frac{\left(1-\frac{2 \alpha_{0}}{2 \pi}\right) l}{A \mu_{2}} \Phi_{02}\right]
\end{aligned}
$$


Where $I_{\mathrm{s}}^{\prime} Z_{\mathrm{s}}^{\prime}$ is the voltage applied on the load $Z_{\mathrm{s}}$ due to the eccentric primary bus bar which can be calculated by (6) and $I_{\mathrm{s}}^{\prime \prime} Z_{\mathrm{s}}^{\prime \prime}$ is the voltage applied on the load $Z_{\mathrm{s}}$ due to the stray flux entering into the core directly induced the currents in the secondary winding. This kind of decomposition could be applied on (2) also, shown in (10). In other words, the measurement error of the HCT affected by the stray flux contains the influence of the eccentric bus-bar on the HCT and the stray flux itself directly induced the currents in the secondary winding.

From (9)-(10), we could see that the crucial parameters of the eccentric bus-bar testing method presented in [1] are of some disadvantage. It is true that the HCT is sensitive to the displacement of the primary bus bar $[6,7]$, but the equivalent eccentric distance which derived from [1] has some problem.

The reason why we use the equivalent testing method to evaluate the reliability of HCTs is that most of the HCT manufacturers and laboratories cannot supply so heavy bus bar currents. The eccentric bus-bar testing method which still uses the original primary current cannot be used in practice.

\section{CONCLUSION}

This paper presents several experiments on the eccentric bus-bar testing method. The results of these experiments reveal some problems of this method. Analytical formulas are used to evaluate the correctness of the crucial parameters of the testing method. The analytical formulas reveal the basic flaw of this method. The practical applicability of this equivalent testing method is analyzed at last.

\section{REFERENCES}

[1] K. Qu, W. Zhao, B. Jiang, S. Huang, Z. Liu, and Q. Dong, "Eccentric bus-bar testing method of the shielding effect of heavy current transformer with shielding coils," in Proc. IEEE Instrum. Meas., Austin, TX, May 3-6, 2010, pp. 721-724.

[2] J. Letosa, A. Uson, J. S. Artal, M. Samplon, F. J. Arcega, "Uncertainties associated with current measurements: Analysis by the finite element method," International Journal of Applied Electromagnetics and Mechanics, 2004, vol. 19, n 1-4, pp. 159-16

[3] H. Y. Yu, J. S Yuan, J. Zou, "Design of novel structure current transformer with shielding coils for overcoming the saturation of core," IEEE Trans Magnetics, 2006, vol. 42, pp. 1431-1434.

[4] R. A. Pfuntner, "The accuracy of current transformers adjacent to heavy current buses," AIEE Trans, 1951, vol. 70, pp. 1656-1661.

[5] S. Seely, "Effect of stray flux on current transformers, "Journal of Science \& Technology, 1970, vol. 37, pp. 115-120.

[6] A. Braun, "Determination of current transformer errors at primary currents up to 100000A," IEEE Trans Instrumentation and Measurement, 1977, vol. 26, pp. 263-267.

[7] J. Letosa, J. S. Artal, M. Samplon, A. Uson, F. J. Arcega, "Modelization of current sensors by finite elements method," Measurement: Journal of the International Measurement Confederation, 2004, vol. 35, n.3, pp. 233-241 